\title{
Brief Report: Autism and Herpes Simplex Encephalitis
}

\author{
Mohammad Ghaziuddin, ${ }^{1}$ Luke Y. Tsai, \\ Laura Eilers, and N. Ghaziuddin \\ Department of Child and Adolescent Psychiatry, University of \\ Michigan Hospital, Ann Arbor
}

\section{INTRODUCTION}

It is now generally accepted that autism is a behavioral syndrome with an underlying organic pathology. It can be associated with a variety of disorders affecting the central nervous system, such as encephalitis. DeLong, Bean, and Brown (1981) described three children, ages between 5 and 11 years, who developed acute encephalitic illness accompanied by autistic features that resolved after clinical recovery. One of the patients had high serum herpes simplex titers, and a computerized tomography (CT) scan of the head revealed an extensive lesion of the temporal lobes, mainly on the left side. The other two patients had normal CT scans, and no etiological agent was defined. Gillberg (1986) described the case of a 14-year-old girl who developed "typical" autistic syndrome following an attack of herpes simplex encephalitis. The CT of the head showed widespread bilateral destruction of brain parenchyma and the temporal lobes, with some medial involvement of the lower parts of the parietal lobes. The autistic symptoms persisted long after the acute symptoms of herpes encephalitis, such as fever and alteration of consciousness, subsided.

All the above cases met the criteria for autism except the typical age of onset. For example, DeLong et al.'s cases ranged in age from 5 to 11

${ }^{1}$ Address all correspondence to Mohammad Ghaziuddin, Division of Child Psychiatry, Taubman Center-Box 0390, University of Michigan Hospitals, 1500 East Medical Center Drive, Ann Arbor, Michigan 48109-0390. 
years when they developed the symptoms, and Gillberg's patient was 14 years old; whereas the onset of autism is usually defined before the age of 3 years. We describe below two children who developed herpes virus infection in the intrauterine or early postnatal period, and presented with features of autism around 2 years of age.

\section{PATIENT 1}

B, a 4-year-old white boy, was referred by his mother for a diagnostic evaluation to rule out autism.

Developmental history revealed that he was born after a full-term normal pregnancy and weighed $10 \mathrm{lb}$ at birth. Problems arose during delivery when meconium staining of the amniotic fluid was noted. The delivery was assisted by forceps. Immediately after birth, B was noted to be tachypneic and was diagnosed as having a "wet lung." He was discharged at 4 days of age with a probable diagnosis of aspiration pneumonitis. His IgM level was 15.7 (normal 6.3 to 25 ); a herpes titer was not done. He was readmitted 10 days later with signs of an upper respiratory infection, vomiting, and a failure to gain weight. He was treated by oxygen and intravenous antibiotics following which he made a slow recovery. A final diagnosis of sepsis neonatorum and chlamydial laryngotracheobronchitis was made. At this time, the titer for TORCH organisms (toxoplasmosis, syphilis, rubella, cytomegalovirus, and herpes virus) was described as negative in the discharge summary. However, according to the pathology report, the herpes titer was read as outside the reference range; it was $1: 4$ whereas the reference range of the laboratory where it was done was less than 1:2. Skull X-ray and CT scan of the head were done and were within normal limits.

B's early motor development was normal. He was able to sit at 6 months of age and could walk without support at $9 \frac{1}{2}$ months. His language development was, however, delayed. He showed no attention span and also indulged in frequent temper tantrums. At 19 months he was evaluated and was found to be functioning at the 8- to 10-month level. Apart from some basic consonant-vowel combinations, his speech was minimal. In addition, his social development was also substantially delayed and inappropriate. For example, he showed no interaction with others and frequently treated people as inanimate objects. He also engaged in self-abuse in the form of head-banging and face-scratching. He never showed any inclination to interact with children of his age. His speech production was limited to making minimal sounds and prolonged screaming. His nonverbal communication, as well as his ability to imitate, were impaired. During psychological testing, 


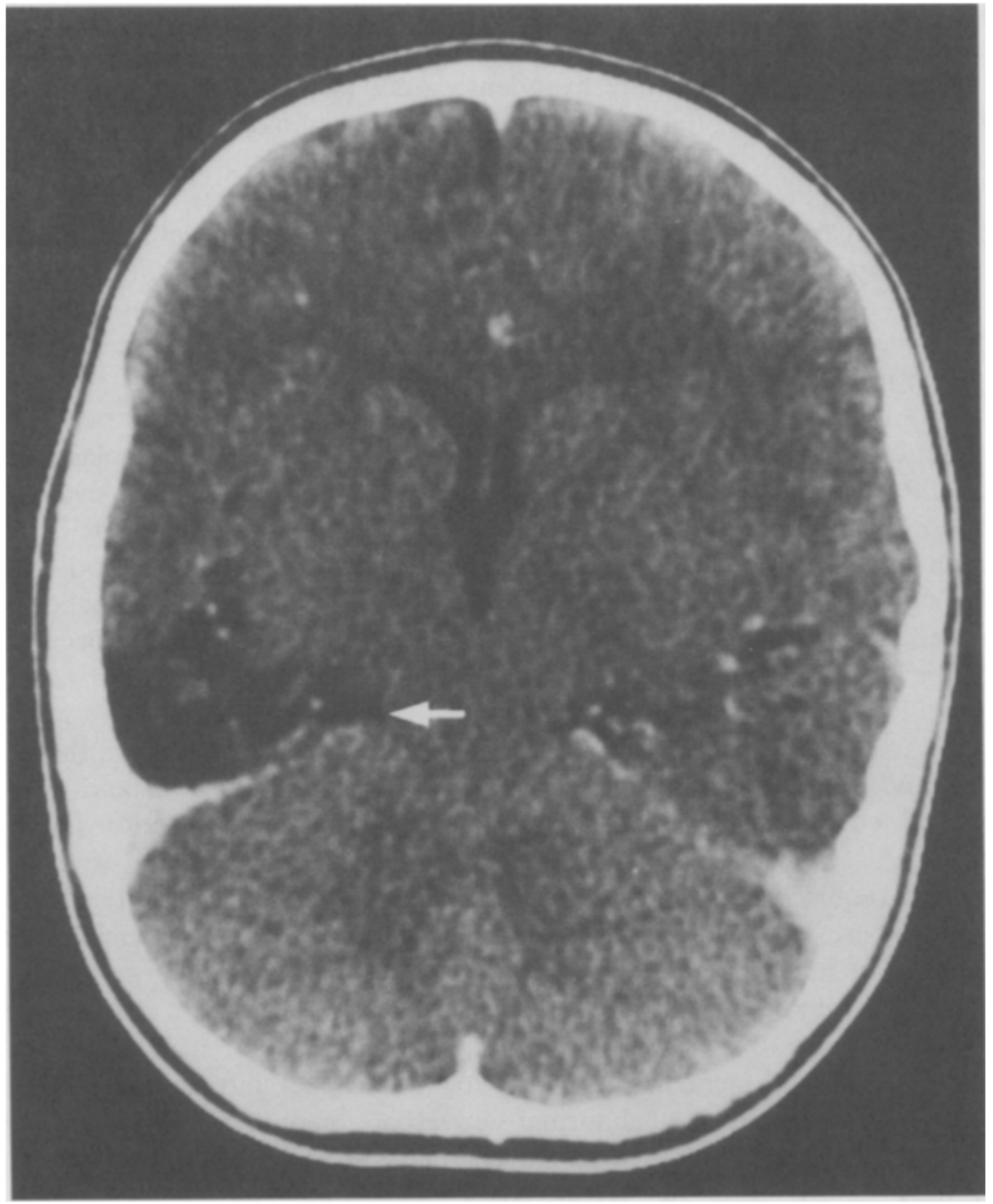

Fig. 1. Head CT Scan of Case 1 showing hypodense areas in the left temporal region.

he showed no representational play or curiosity of object use. It was not possible to give a score to his IQ level because of his problems with attention and concentration. However, on the basis of clinical observation, his IQ was placed below 50 .

At 2 years of age, $B$ received a neurological evaluation to assess the possibility of a seizure disorder and was, in fact, placed on carbamazepine. 
He had a history of 2- to 3-second periods of rolling back his eyes with fluttering of eyelids. An EEG showed no evidence of seizures. A CT scan of the head showed definite hypodense areas in the temporal regions on both sides, more on the left. According to the radiologist, the area of attenuation on the left and involvement of the contralateral temporal lobe in nearly identical topography was unusual for a vascular, metabolic, or ischemic reason. The most likely cause was felt to be inflammatory, especially herpes simplex encephalitis. Other features consistent with this diagnosis were the overall slight decrease in the brain volume for age and the presence of some calcification along the pulvinar of the thalamus. This is shown in the CT scan picture (Figure 1).

At the same time, a repeat of the TORCH titers revealed persisting antibodies to herpes virus but none to cytomegalovirus and toxoplasmosis. On the basis of the positive antibody titer to herpes virus and the evidence of cortical damage on CT scan of the head, it was concluded that B had been infected with herpes simplex either in the intrauterine period or in the postnatal period. The lack of clinical evidence of herpes infection in either parent left the possibility that the infection had probably occurred in the neonatal period although the exact time of infection could not be identified in retrospect.

So far as the behavioral symptoms were concerned, based on the history and the collateral evaluations, B met the criteria for autistic disorder as defined in the DSM-III-R (American Psychiatric Association [APA], 1987). Also, his aunt and his mother rated him on at least 8 of the 16 criteria required for the diagnosis of autistic disorder based on the DSMIII-R. These included his qualitative impairment in reciprocal social interaction and impairments in language and play even when his mental age was taken into consideration. Some of his behaviors included marked lack of awareness of the existence of others, impaired imitation, lack of social play, gross impairment in the ability to initiate peer interaction, minimal mode of communication, abnormal nonverbal communication, absence of imaginative activity, stereotyped movements, and so forth. On the modified Autism Behavior Checklist (Krug, Arick, \& Almond, 1980), his scores were 84 and 96 given by his aunt and mother, respectively, which further strengthened the diagnosis of autistic disorder. In addition, he suffered from mental retardation.

\section{PATIENT 2}

$\mathrm{S}$, an 11-year-old boy with severe mental retardation, was referred for a diagnostic evaluation and management of his behavioral symptoms. 
These consisted of frequent temper tantrums, self-injurious behavior, and long periods of spinning and rocking. His eye contact was minimal and inappropriate. He had little verbal speech which consisted of grunting sounds with a tendency to persistent repetition.

He was the product of a full-term normal delivery. The perinatal period was uncomplicated; however, on the 11th day, mother noted that he did not wake with normal frequency during the night; also, his oral intake had markedly decreased. On examination, he was lethargic but arousable. A spinal tap showed elevated white cells and protein. Skull $\mathrm{X}$-ray was normal but a CT scan was not done. TORCH screen was negative. On the 17th day, a small vesicle on the right eyelid was noted, which was sent for viral culture. Three days later, he was noted to have tonic-clonic movements of his left arm and leg with deviation of the eyes to the left. Results of the vesical culture showed herpes simplex. An EEG showed paroxysmal discharges, maximal on the left side. In addition, an ophthalmologic consultation revealed bilateral corneal staining with bilateral uveitis. He later developed seizures and was treated with anticonvulsants. On the basis of these findings, a diagnosis of herpes simplex encephalitis was made for which he was started on a course of arabinoside. Throughout the hospital course, he showed general improvement in feeding and activity. A repeat spinal tap showed a decrease in white blood cells and protein, and he was discharged home on arabinoside eye ointment and phenobarbitone. The discharge diagnosis was herpes encephalitis and keratouveitis.

As S grew up, he was noted to be "socially different." He tended to ignore his surroundings, including the members of his family, and did not reach out to be picked up. Occasionally, he showed no startle response to loud noises. He walked at about 12 months of age and initially insisted on walking only on toes. He began saying single words, such as mom, dad, and so forth, around 2 years of age, but word usage gradually diminished to undifferentiated sounds which was his level of communication at the time of referral. At about the age of 3-4 years, he was place in a program for mentally retarded children but his behavioral symptoms continued to increase. Thus, at 7-8 years of age additional services were recommended prior to the referral to our clinic.

Based on the above findings, a DSM-III-R diagnosis of autistic disorder (APA, 1987) with mental retardation, was made. The criteria in favor of this diagnosis were a marked lack of awareness of the existence of others, sometimes treating people as inanimate objects; absence of imitation and lack of eye contact; absence of social play and interaction, even when his mental retardation was taken into account; presence of stereotyped movements such as spinning and flapping etc; marked abnormalities in the form 
and content of speech; and absence of any social or imaginative play; and strong reactions to changes in the environment.

\section{DISCUSSION}

These case reports document the occurrence of autistic disorder in two children with a past history of herpes encephalitis. The exact time of occurrence of the infection was not clear; it was probably in the intrauterine or the early postnatal period. The infection was demonstrated by the presence of antibody titers and the involvement of the temporal lobes on the CT scan of the head in the first patient. In the second patient, involvement of the central nervous system was suggested by altered consciousness, tonic-clonic movements of the left side of the body, electroencephalographic changes, and abnormalities on the spinal tap. This was strongly suspected to be herpetic in origin because of the positive vesical culture and the characteristic involvement of the eyes.

Greer, Lyons-Crews, Mauldin, and Brown (1989) recently commented on the cognitive and behavioral deficits of temporal lobe damage in herpes encephalitis. They described a 14-year-old boy, who was apparently normal till the second grade when he was admitted to hospital with herpes simplex encephalitis. He later developed significant and persistent language, social, and memory deficits. Herpes virus appears to have a predilection for the temporal lobes (Greer et al., 1989). Several studies have implicated the temporal lobes in the pathogenesis of autism (e.g., Heltzer \& Griffin, 1981). Jones and Kerwin (1990), for instance, described a patient with Asperger syndrome, widely regarded as a mild variant of autism, who showed left temporal lobe damage on computerized tomography. This does not imply, however, that temporal lobe pathology is commonly associated with autism. In fact, a variety of lesions in the brain have been documented as associated with autism, in particular the cerebellum (see Courchesne, 1991).

Despite the evidence pointing to the involvement of temporal lobes in herpes encephalitis, no causative role for the virus in the etiology of the disorder has been established. The report by DeLong et al. (1981) suggested that herpes encephalitis may cause reversible autistic-like symptoms, whereas Gillberg's (1986) report suggested that herpes simplex virus-induced autism may result even at the age of 14 years. To our knowledge, no one has yet reported any case of autism with such a late age of onset. Both cases described in this report had an early history of herpes simplex encephalitis as well as of autism. This is important because symptoms arising from the failure of development of temporal lobes in early life may be different from those arising from late destruction of previously normal 
lobes. This report adds further support to the hypothesis that herpes simplex encephalitis, possibly through the involvement of temporal lobes, is associated with autism but does not propose a direct cause-and-effect relationship between the two. A systematic investigation of the cognitive and behavioral deficits of children with an early history of herpes virus encephalitis, using a large number of patients, is needed to further explore the nature of this association.

\section{REFERENCES}

American Psychiatric Association. (1987). Diagnostic and statistical manual of mental disorders (3rd ed., rev.). Washington, DC: Author.

Courchesne, E. (1991). Neuroanatomic imaging in autism. Pediatrics, 87 (Suppl. part 2), 781890.

DeLong, G. R., Bean, S. C., \& Brown, F. R., III. (1981). Acquired reversible autistic syndrome in acute encephalopathic illness in children. Archives of Neurology, 38, 191, 194.

Gillberg, C. (1986). Brief report: Onset at age 14 of a typical autistic syndrome. A case report of a girl with herpes simplex encephalitis. Joumal of Autism and Developmental Disorders, 16, 369-375.

Greer, M. K., Lyons-Crews, M., Mauldin, L. B., \& Brown, F. R., III. (1989). A case study of the cognitive and behavioral deficits of temporal lobe damage in herpes simplex encephalitis. Journal of Autism and Developmental Disorders, 19, 317-326.

Hetzler, B., \& Griffin, J. (1981). Infantile autism and the temporal lobe of the brain. Journal of Autism and Developmental Disorders, 11, 317-330.

Jones, P. B., \& Kerwin, R. W. (1990). Left temporal lobe damage in Asperger's syndrome. British Journal of Psychiatry, 156, 570-572.

Krug, D. A., Arick, J. R., \& Almond, P. J. (1980). Behavior checklist for identifying severely handicapped individuals with high levels of autistic behavior. Joumal of Child Psychology and Psychiatry, 21, 221-229. 